\title{
Caracterização molecular e variabilidade genética de acessos elite de mandioca para fins industriais
}

\author{
Molecular characterization and genetic variability of elite cassava \\ accessions for industrial purpose
}

\begin{abstract}
Eduardo Alano Vieira ${ }^{I^{*}}$ Josefino de Freitas Fialho $^{\mathrm{I}}$ Fábio Gelape Faleiro ${ }^{\mathrm{I}}$ Graciele Bellon $^{\mathrm{I}}$ Kenia Gracielle da FonsecaI Luiz Joaquim Castelo Branco Carvalho ${ }^{\text {II }}$ Marilia Santos Silva
\end{abstract}

\section{RESUMO}

Marcadores moleculares são ferramentas úteis na caracterização molecular de acessos de mandioca, em razão de apresentarem elevada capacidade de detecção das informações contidas no genoma. O objetivo deste trabalho foi caracterizar, por meio de marcadores RAPD, 20 acessos de mandioca para fins industriais conservados no Banco Regional de Germoplasma de Mandioca do Cerrado (BGMC). Em laboratório, os acessos foram avaliados por meio de marcadores RAPD, sendo posteriormente estimada a matriz de similaridade genética entre os acessos, por meio do indice de Jaccard. A análise, feita através de 11 iniciadores, gerou um total de 120 marcadores RAPD, dos quais 74 (62\%) foram polimórficos, revelando a presença de elevada variabilidade genética no grupo de acessos avaliados. A análise de agrupamento revelou a formação de apenas um agrupamento forte, formado pelos acessos BGMC 1130, BGMC 788, BGMC 1270 e $B G M C 1107$, o que indica que, no melhoramento genético de mandioca, não devem ser priorizadas hibridações entre esses acessos, sob pena de efeitos de endogamia. Por sua vez, o acesso BGMC 436 foi o mais divergente em relação aos demais e, como expressa elevado potencial produtivo na região do Cerrado do Brasil Central, representa boa opção como genitor para o melhoramento de mandioca para essa região. $O$ estudo comprovou que os marcadores RAPD são eficientes na determinação da variabilidade genética dos acessos avaliados e que neste grupo existe elevada variabilidade genética passivel de ser utilizada no melhoramento genético.

Palavras-chave: Manihot esculenta Crantz, recursos genéticos, melhoramento genético, fécula.

\section{ABSTRACT}

Molecular markers are useful tools for the molecular characterization of cassava accessions since they present high capacity to detect information within the genome. The aim of this research was to characterize through RAPD molecular markers, 20 industrial cassava accessions conserved in the Cerrado Cassava Regional Germoplasm Bank ("Banco Regional de Germoplasma de Mandioca do Cerrado"-BGMC). Upon laboratory conditions, the accessions were evaluated though RAPD markers, being afterwards estimated by the matrix of genetic similarity among the accessions, through the Jaccard index. The analyses through 11 primers generated a total of 120 RAPD markers, among which 74 (62\%) were polymorphic, revealing the presence of high genetic variability within the group of evaluated accessions. The clustering analyses revealed the formation of a single strong group, comprised of the accessions BGMC 1130, BGMC 788, BGMC 1270 and BGMC 1107, which indicates that the cassava genetic breeding hybridizations among these accessions should not be prioritized, upon risk of endogamy effects. The accession BGMC 436 was the most divergent as compared to the others and, as it expresses high productive potential within the Central Brazil Cerrado, it represents a good option as parent for the cassava breeding in this region. The study confirmed that the RAPD markers are efficient for the determination of genetic variability among evaluated accessions and that within the group there is high genetic variability useful for the plant breeding.

Key words: Manihot esculenta Crantz, genetic resources, plant breeding, starch.

\section{INTRODUÇÃO}

A mandioca (Manihot esculenta Crantz) foi provavelmente domesticada na região de transição entre o Cerrado e a Amazônia, há cerca de seis mil anos, em razão de suas raízes tuberosas ricas em amido e

IEmbrapa Cerrados, BR 020, Km 18, 73310-970, Planaltina, DF, Brasil. E-mail: vieiraea@cpac.embrapa.br. "Autor para correspondência.

"Embrapa Cenargen, Brasília, DF, Brasil. 
amplamente utilizadas na alimentação das populações pré-colombianas (OLSEN, 2005; CARVALHO, 2005). No Brasil, a cultura é cultivada de Norte a Sul, revelando elevada capacidade de adaptação as mais diversas condições ambientais, desde o clima tropical úmido da Amazônia até o clima subtropical do Rio Grande do Sul. A cultura é cultivada principalmente visando à alimentação humana, na forma de fécula, farinha e in natura (cozida e/ou frita). Também pode ser utilizada na alimentação animal (parte aérea e/ou raízes) e na indústria química, metalúrgica, têxtil, farmacêutica, plástica, entre outras (CARDOSO \& SOUZA, 2002).

No Brasil, a produção de fécula, derivado de mandioca com maior potencial de comercialização no mercado nacional e internacional, está concentrada nos Estados do Paraná, São Paulo e Mato Grosso do Sul. Entretanto, nos últimos anos, vêm crescendo a demanda dos produtores por variedades de mandioca de interesse para a indústria, específicas para o Cerrado do Brasil Central, como consequência da instalação de novas fecularias na região e da perspectiva de mercado criada(VILPOUX, 2008).

Nesse contexto, fica evidente a necessidade de realização de melhoramento genético nessa espécie, visando à seleção de constituições genéticas específicas para a região do Cerrado do Brasil Central. Entretanto, para que o melhoramento genético seja eficiente, é necessário conhecimento a cerca da variabilidade genética existente na coleção de trabalho, da qual serão selecionados os genitores a serem utilizados na geração das populações híbridas, o que facilitará sobremaneira a tomada de decisão a cerca da utilização e da conservação do germoplasma disponível.

$\mathrm{Na}$ cultura da mandioca, marcadores moleculares vêm sendo utilizados com elevada frequência na estimativa da variabilidade genética, em razão de apresentarem elevada capacidade de detecção das informações contidas no genoma (ZACARIAS et al., 2004; FERREIRA et al., 2008, VIEIRA et al., 2008a). Dentre as diversas técnicas de marcadores moleculares, o polimorfismo de DNA amplificado ao acaso (RAPD) merece destaque, pelo baixo custo, pela rápida e fácil execução e pelo fato de não exigir a síntese de iniciadores específicos.

Neste trabalho, objetivou-se caracterizar, por meio de marcadores RAPD, 20 acessos de mandioca de interesse para a indústria conservados no Banco Regional de Germoplasma de Mandioca do Cerrado (BGMC).

\section{MATERIAL E MÉTODOS}

Neste estudo, foram analisados vinte acessos elite de mandioca de interesse para a indústria mantidos no Banco Regional de Germoplasma de Mandioca do Cerrado (BGMC), sendo quatro desenvolvidos pelo programa de melhoramento genético de mandioca do Instituto Agronômico de Campinas (IAC), oito pelo programa de melhoramento genético de mandioca da Embrapa Mandioca e Fruticultura Tropical (CNPMF) e oito variedades locais, selecionadas em razão de apresentarem potencial de adaptação às condições do Cerrado do Brasil Central como variedades para indústria (Tabela 1) (VIEIRA et al., 2008b).

Amostras de DNA para obtenção dos marcadores RAPD foram obtidas a partir de $5 \mathrm{~g}$ de folhas em estágio intermediário de maturação, utilizando o método do brometo de cetiltrimetilomônio (CTAB), com pequenas modificações (FALEIRO et al., 2003). O DNA foi quantificado por espectrofotometria a $260 \mathrm{~nm}$ e a relação A260/A280 foi utilizada para avaliar a pureza do DNA.

As reações de amplificação foram realizadas em um volume total de $13 \mu \mathrm{L}$, contendo Tris- $\mathrm{HCl} 10 \mathrm{mM}$ (pH 8,3), $\mathrm{KCl} 50 \mathrm{mM}, \mathrm{MgCl} 23 \mathrm{mM}, 100 \mu \mathrm{M}$ de cada um dos desoxiribonucleotídios (dATP, dTTP, dGTP e $\mathrm{dCTP}), 0,4 \mu \mathrm{M}$ de um iniciador, uma unidade da enzima Taq polimerase e $15 \mathrm{ng}$ de DNA. Na obtenção dos marcadores RAPD, foram utilizados 11 iniciadores

Tabela 1 - Acessos de mandioca de indústria analisados com respectivos nomes comuns e locais de coleta/procedência (LCP).

\begin{tabular}{lll}
\hline Acessos & \multicolumn{1}{c}{ Nome comum } & \multicolumn{1}{c}{ LCP } \\
\hline BGMC 436 & IAC 12 & IAC - São Paulo* \\
BGMC 1130 & IAC 13 & IAC - São Paulo* \\
BGMC 788 & IAC 14 & IAC - São Paulo* \\
BGMC 1270 & IAC 15 & IAC - São Paulo* \\
BGMC 1304 & Clone 9661/06 & CPMF - Bahia** \\
BGMC 1107 & Fécula Branca & Paraná \\
BGMC 1286 & Roxinha & Santa Catarina \\
BGMC 1297 & BRS Mani-Branca & CPMF - Bahia** \\
BGMC 1305 & Clone 9688/07 & CPMF - Bahia** \\
BGMC 1298 & Clone 9794/06 & CPMF - Bahia** \\
BGMC 991 & Clone 03 & CPMF - Bahia \\
BGMC 923 & BRS Formosa & CPMF - Bahia \\
BGMC 1356 & Piriquita & Minas Gerais \\
BGMC 996 & Clone 09 & CPMF - Bahia** \\
BGMC 1299 & CNPMF 9607/7 & CPMF - Bahia** \\
BGMC 1265 & Fibra & Paraná \\
BGMC 1262 & Enita Brava & Tocantins \\
BGMC 1371 & Araras Pecíolo Vermelho & Minas Gerais \\
BGMC 1372 & Araras Pecíolo Amarelo & Minas Gerais \\
BGMC 56 & Branca de Santa Catarina & Santa Catarina \\
\hline
\end{tabular}

*=Programa de melhoramento genético de mandioca do Instituto Agronômico de Campinas (IAC), baseado em Campinas (SP);

** Programa de melhoramento genético de mandioca da Embrapa Mandioca e Fruticultura Tropical, baseado em Cruz das Almas (BA). 
decâmeros da série Operon (Operon Biotechnologies): OPD (02), OPE (12), OPF (08), OPG (05, 08, 09, 15 e 16), $\mathrm{OPH}(4,13$ e 15$)$. Na etapa de amplificação dos segmentos de DNA, foi utilizado termociclador programado para 40 ciclos, cada um constituído pela seguinte sequência: 15 segundos a $94^{\circ} \mathrm{C}, 30$ segundos a $35^{\circ} \mathrm{C}$ e 90 segundos a $72^{\circ} \mathrm{C}$. Após os 40 ciclos, foi realizada uma etapa de extensão final de seis minutos a $72^{\circ} \mathrm{C}$ e, posteriormente, a temperatura foi reduzida para $4^{\circ} \mathrm{C}$. Após a amplificação, foram adicionadas, a cada amostra, $3 \mu 1$ de uma mistura de azul de bromofenol $(0,25 \%)$ e glicerol $(60 \%)$ em água. Essas amostras foram aplicadas em gel de agarose $(1,2 \%)$, corado com brometo de etídio, submerso em tampão TBE (TrisBorato $90 \mathrm{mM}$, EDTA $1 \mathrm{mM}$ ). Os produtos amplificados foram separados eletroforeticamente e fotografados sob luz ultravioleta.

Os produtos das reações de amplificação (marcadores RAPD) foram classificados em presença (1) e ausência (0) de bandas e convertidos em uma matriz de dados binários, a partir da qual foi estimado: i) o conteúdo de informação de polimorfismo (CIP) de cada marcador de cada iniciador, por meio da fórmula $\mathrm{CIP}=2 \mathrm{f}_{\mathrm{i}}\left(1-\mathrm{f}_{\mathrm{i}}\right)$, em que fi é a frequência de presença de banda (1), 1-fi é a frequência de ausência de banda (0), e posteriormente foi estimado o CIP médio de cada iniciador por meio da média aritmética do CIPs obtidos; ii) o índice do marcador (IM), calculado para cada iniciador por meio da fórmula IM=PIC x nbp, em que npb é o número de bandas polimórficas (POWELL et al., 1996); e iii) a similaridade genética entre os acessos, por meio do coeficiente de Jaccard (JACCARD, 1908), conforme a equação: $\mathrm{S}_{\mathrm{ij}}=\mathrm{N}_{\mathrm{ij}} /\left(\mathrm{N}_{\mathrm{ij}}+\mathrm{N}_{\mathrm{i}}+\mathrm{N}_{\mathrm{j}}\right)$, em que: $\mathrm{N}_{\mathrm{ij}}=$ número de bandas presentes em ambos os acessos i e j; $\mathrm{N}_{\mathrm{i}}=\mathrm{o}$ número de bandas presentes no acesso $\mathrm{i} ; \mathrm{e}_{\mathrm{j}}=\mathrm{o}$ número de bandas presentes no acesso $\mathrm{j}$.

Com base na matriz de similaridade, foi construído um dendrograma por meio do método de agrupamento da distância média (UPGMA). $\mathrm{O}$ ajuste entre a matriz de dissimilaridade e o dendrograma foi estimado pelo coeficiente de correlação cofenética (r), conforme SOKAL \& ROHLF (1962), por meio do programa computacional NTSYS pc 2.1 (ROHLF, 2000). A estabilidade dos agrupamentos foi estimada por meio da análise de bootstraping com 500 replicações, por meio do programa Genes (CRUZ, 2001).

\section{RESULTADOS E DISCUSSÃO}

A análise de 20 acessos de mandioca de interesse para a indústria por meio de 11 iniciadores gerou um total de 120 marcadores RAPD, dos quais 74 (62\%) foram polimórficos (Tabela 2). A elevada eficiência da técnica de marcadores RAPD na discriminação de acessos de mandioca já havia sido relatada por COLOMBO et al. (2000); ASANTE \& OFFEI, (2003);
ZACARIAS et al., (2004); e VIEIRA et al. (2008a), que encontraram porcentagens de polimorfismo de $46 \%$, $97,5 \%, 84,6 \%$ e $73 \%$, respectivamente. Os iniciadores que evidenciaram mais bandas polimórficas (BP), maiores conteúdos de informação de polimorfismo (CIP) e maiores informações de polimorfismo (MI) foram OPG15 e OPF-08, com, respectivamente, 15 e 14 (BP); 0,27 e 0,21 (CIP); e 4,05 e 2,94 (MI) (Tabela 2). Por outro lado, $\mathrm{o}$ único iniciador que não revelou polimorfismo entre os acessos avaliados foi o OPH-13 (Tabela 2).

A matriz similaridade genética revelou que os acessos mais similares foram BGMC 1286 (variedade local procedente do Estado de Santa Catarina) e BGMC 1297 (variedade melhorada recomendada para o cultivo no nordeste do Brasil) (Figura 1) e que os acessos que revelaram a menor similaridade genética entre si foram BGMC 1270 (IAC 15 variedade melhorada recomendada para o cultivo na região centro-sul do Brasil) e BGMC 1371 (variedade local procedente do Estado de Minas Gerais) (matriz de similaridade não apresentada).

A análise da figura 1 revelou a formação de apenas dois agrupamentos fortes, com mais de $70 \%$ de agrupamentos coincidentes, sendo um formado pelos acessos BGMC 1130, BGMC 788, BGMC 1270 e BGMC 1107 com uma percentagem de agrupamento coincidente de $75 \%$ (Figura 1). Dentre os acessos do referido grupo, três foram desenvolvidos pelo programa de melhoramento genético do IAC e um é a variedade procedente do Estado do Paraná, conhecida popularmente como Fécula Branca (Tabela 1). Do ponto de vista prático, esse cenário indica que, no melhoramento genético de mandioca de indústria, não devem ser priorizadas hibridações entre esses acessos, sob pena de efeitos de endogamia, que, segundo

Tabela 2 - Iniciadores utilizados para obtenção dos marcadores RAPD, respectivo número de bandas polimórficas (BP), número de bandas monomórficas (BM), conteúdo de informação de polimorfismo (CIP) e indíce do marcador (IM).

\begin{tabular}{llllll}
\hline Iniciadores & $\begin{array}{c}\text { Sequência } \\
5^{\prime} \rightarrow 3^{\prime}\end{array}$ & BP & BM & CIP & IM \\
\hline OPD-02 & GGACCCAACC & 5 & 3 & 0,16 & 0,80 \\
OPE-12 & TTATCGCCCC & 9 & 4 & 0,26 & 2,34 \\
OPF-08 & GGGATATCGG & 14 & 1 & 0,21 & 2,94 \\
OPG-05 & CTGAGACGGA & 6 & 5 & 0,17 & 1,02 \\
OPG-08 & TCACGTCCAC & 6 & 4 & 0,11 & 0,66 \\
OPG-09 & CTGACGTCAC & 8 & 5 & 0,25 & 2,00 \\
OPG-15 & ACTGGGACTC & 15 & 3 & 0,27 & 4,05 \\
OPG-16 & AGCGTCCTCC & 4 & 4 & 0,13 & 0,52 \\
OPH-04 & GGAAGTCGCC & 3 & 7 & 0,06 & 0,18 \\
OPH-13 & GACGCCACAC & 0 & 5 & 0,00 & 0,00 \\
OPH-15 & AATGGCGCAG & 4 & 5 & 0,08 & 0,32 \\
TOTAL & & 74 & 46 & 0,15 & 1,35 \\
\hline
\end{tabular}




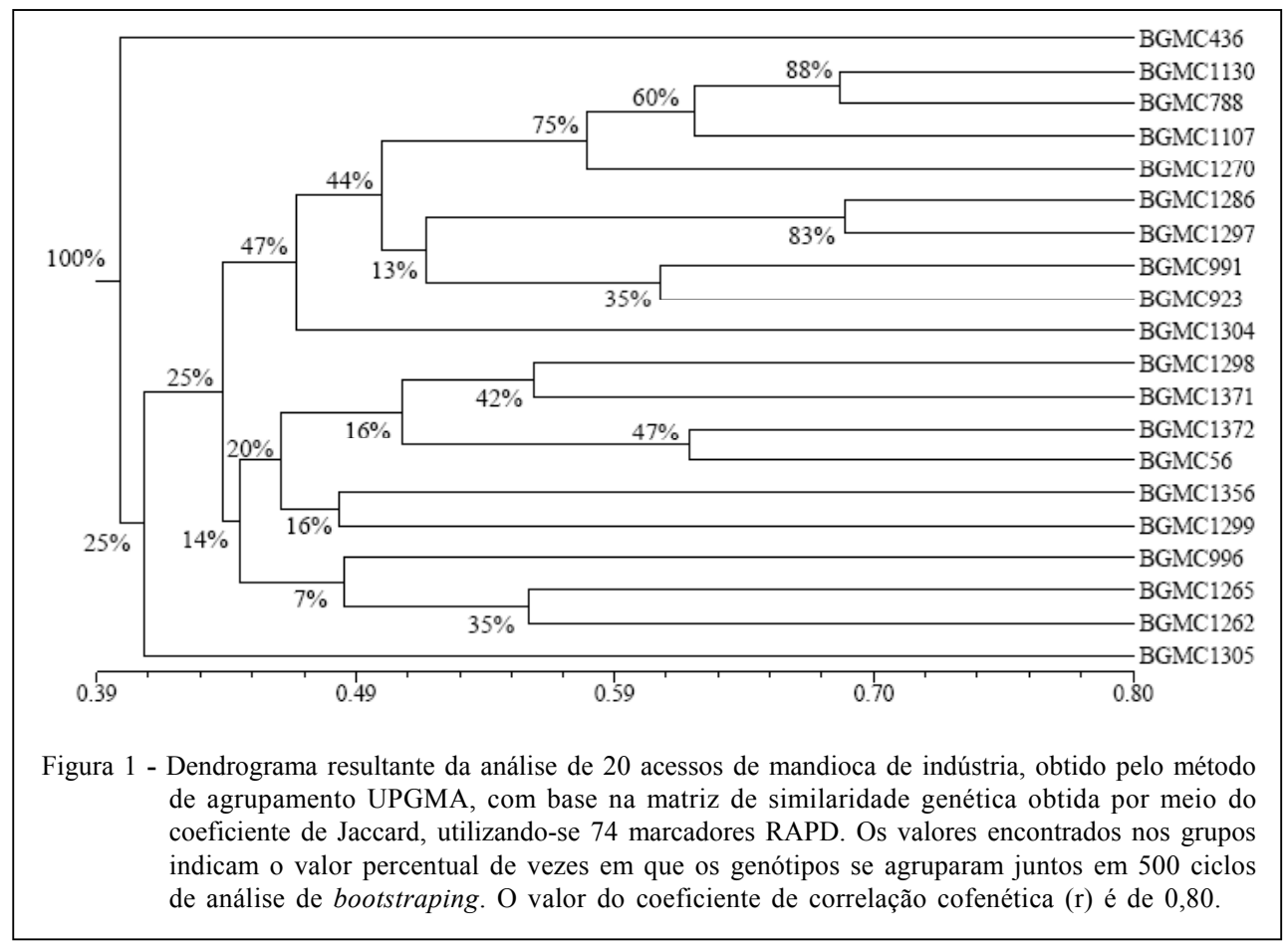

CEBALLOS et al. (2004), são muito pronunciados em mandioca. Por sua vez, o acesso BGMC 436, que também foi desenvolvido pelo IAC, foi o mais divergente em relação aos demais (Tabela 1 e Figura 1) e, como expressa elevado potencial produtivo na região do Cerrado do Brasil Central (VIEIRA et al., 2008a), representa boa opção como genitor para o melhoramento de mandioca para essa região, para cruzamentos com os demais acessos avaliados. Outro agrupamento forte com uma porcentagem de agrupamento coincidente de $83 \%$ foi formado pelos acessos BGMC 1286 (variedade local de Santa Catarina) e BGMC 1297 (variedade melhorada, recomendada para o cultivo no nordeste do Brasil) (Figura 1). O coeficiente de correlação cofenética do dendrograma $(\mathrm{r}=0,80)$ revelou elevado ajuste entre a representação gráfica da similaridade genética e a matriz de similaridade original, o que justifica que sejam realizadas inferências por meio da avaliação visual da figura 1 .

Os demais acessos avaliados não apresentaram tendência de agrupamento de forma hierárquica, segundo a análise de bootstraping (Figura 1). Fato interessante foi a inexistência de tendência de agrupamento dos acessos gerados pelo programa de melhoramento genético da Embrapa Mandioca e Fruticultura Tropical (Tabela 1, Figura 1). Provavelmente, a ausência de agrupamentos coincidentes decorra do fato de esse programa de melhoramento gerar constituições genéticas para diversas regiões do nordeste brasileiro que apresentam características ambientais contrastantes (FUKUDA et al., 2006) e que, como consequência, exigem que seja utilizada uma ampla base genética no melhoramento.

Os resultados obtidos revelaram claramente a existência de ampla divergência genética no grupo de acessos estudados, o que é reflexo direto do fato de terem sido analisados acessos de diferentes origens e níveis de melhoramento, todos oriundos do Brasil, que é o centro de origem e diversidade da espécie (OLSEN, 2004). Essa elevada variabilidade genética aponta para a possibilidade de também haver variação quanto ao potencial agronômico no grupo de acessos avaliados, e que essa variação pode ser utilizada no melhoramento genético de mandioca.

A existência de elevada variabilidade em mandioca, entre acessos oriundos do melhoramento genético, pode ser explicada pela ampla base genética disponível em bancos de germoplasma no Brasil (VIEIRA et al., 2008b) e que é utilizada constantemente no melhoramento da cultura (FUKUDA et al., 2006). A existência dessa ampla variabilidade em mandioca pode ser explicada pelo fato de a espécie ser alógama (ELSHARKAWY, 2004), permitindo que constantemente sejam geradas naturalmente novas combinações, as quais são inicialmente propagadas por sementes e posteriormente propagadas vegetativamente, como uma nova variedade ou incorporadas a uma variedade já cultivada em função do interesse do produtor 
(CEBALLOS et al., 2004; DUPUTIÉ et al., 2009). Ou seja, a mandioca combina a reprodução sexual, que permite à espécie uma constante adaptação às mudanças ambientais e à reprodução vegetativa, que permite uma adaptação imediata às condições ambientais por meio da clonagem de genótipos superiores.

\section{CONCLUSÃO}

Os marcadores RAPD mostraram eficiência na caracterização molecular e determinação da variabilidade genética entre acessos de mandioca de interesse para a indústria. No grupo de acessos avaliados, existe elevada variabilidade genética, passível de ser utilizada no melhoramento genético.

\section{AGRADECIMENTOS}

Os autores agradecem à Embrapa, à Fundação Banco do Brasil, ao Conselho Nacional de Pesquisa (CNPq) e ao Programa Biodiversidade Brasil-Itália (PBBI), pelo apoio financeiro.

\section{REFERÊNCIAS}

ASANTE, I.K.; OFFEI, S.K. RAPD-based genetic diversity study of fifty cassava (Manihot esculenta Crantz) genotypes. Euphytica, v.131, p.113-119, 2003. Disponível em: <http:// www.springerlink.com/content/x $401443488765600 /$ fulltext.pdf $>$. Acesso em: 11 jan. 2010. doi: 10.1023/ A: 1023056313776 .

CARDOSO, C.E.L.; SOUZA, J.S. Importância, potencialidades e perspectivas do cultivo da mandioca na América Latina. In: CEREDA, M.P. Culturas de tuberosas amiláceas latino americanas. Campinas: Fundação CarCargill, 2002. p.2947.

CARVALHO, L.J.C.B. Biodiversidade e biotecnologia em mandioca (Manihot esculenta Crantz). In: CONGRESSO BRASILEIRO DE MANDIOCA, 11., 2005, Campo Grande, MS. Resumos... Campo Grande : Embrapa Agropecuária Oeste, 2005. Não paginado.

CEBALLOS, $\mathrm{H}$. et al. Cassava breeding: opportunities and challenges. Plant Molecular Biology, v.56, p.503-516, 2004. Disponível em: <http://www.springerlink.com/content/p7j212127404664j/ fulltext.pdf>. Acesso em: 11 jan. 2010. doi: 10.1007/s11103-0045010-5.

COLOMBO, C. et al. Diversity within American cassava germplasm based on RAPD markers. Genetics and Molecular Biology, v.31, p.189-199, 2000. Disponível em: <http:// www.scielo.br/pdf/gmb/v23n1/2331.pdf>. Acesso em: 11 jan. 2010. doi: 10.1590/S1415-47572000000100034.

CRUZ, C.D. Programa genes: aplicativo computacional em genética e estatística. Viçosa: UFV, 2001. 648p.

DUPUTIÉ, A. et al. Traditional Ameridian cultivators combine directional and ideotypic selection for sustaineble management of cassava genetic diversity. Journal of Evolutionary Biology, v.22, p.1317-1325, 2009. Disponível em: <http://www3.interscience.wiley.com/cgi-bin/fulltext/ 122352728/PDFSTART $>$. Acesso em: 11 jan. 2010. doi: 10.1111/j.1420-9101.2009.01749.

EL-SHARKAWY, M.A. Cassava biology and physiology. Plant Molecular Biology, v.56, p.481-501, 2004. Disponível em: $<$ http://www.springerlink.com/content/h818787333327r78/ fulltext.pdf $>$. Acesso em: 11 jan. 2010. doi: 10.1007/s11103005-2270-7.

FALEIRO, F.G. et al. Metodologia para operacionalizar a extração de DNA de espécies nativas do cerrado. Planaltina: CPAC, 2003. 6p. (Comunicado Técnico, 92).

FERREIRA, C.F. et al. Molecular characterization of cassava (Manihot esculenta Crantz) with yellow-orange roots for betacarotene improvement. Crop Breeding and Applied Biotechnology, v.8, p.23-29, 2008. Disponível em: <http:// www.sbmp.org.br/cbab/siscbab/uploads/bd6b9df0-119262d6.pdf>. Acesso em: 11 jan. 2010.

FUKUDA, W.M.G. et al. Variedades. In: SOUSA, L.S. et al. Aspectos socioeconômicos e agronômicos da mandioca. Cruz das Almas: Embrapa Mandioca e Fruticultura Tropical, 2006. p.433-454.

JACCARD, P. Nouvelles recherches sur la distribution florale. Bulletin Société Vaudoise des Sciences Naturelles, v.44, p.223-270, 1908.

OLSEN, K.M. SNPs, SSRs and inferences on cassava's origin. Plant Molecular Biology, v.56, p.517-526, 2004. Disponível em: <http://www.springerlink.com/content/v1j742p484403748/ fulltext.pdf $>$. Acesso em: 11 jan. 2010. doi: 10.1007/s11103004-5043-9.

POWELL, W. et al. The comparison of RFLP, RAPD, AFLP and SSR (microsatellite) markers for germplasm analysis. Molecular Breeding, v.2, p.225-238, 1996.

ROHLF, F.J. NTSYS-pc: numerical taxonomy and multivariate analysis system, version 2.1. New York: Exeter Software, 2000. 98p.

SOKAL, R.R.; ROHLF, F.J. The comparison of dendrograms by objective methods. Taxon, v.11, p.30-40, 1962.

VIEIRA, E.A. et al. Divergência genética entre acessos açucarados e não açucarados de mandioca. Pesquisa Agropecuária Brasileira, v.43, p.1707-1715, 2008a. Disponível em: <http:/ /www.scielo.br/pdf/pab/v43n12/v43n12a10.pdf>. Acesso em: 11 jan. 2010. doi: 10.1590/S0100-204X2008001200010.

VIEIRA, E.A. et al. Variabilidade genética do banco de germoplasma de mandioca da Embrapa Cerrados acessada por meio de descritores morfológicos. Científica, v.36, p.56-67, 2008b. Disponível em: < http://www.cientifica.org.br/index.php/ cientifica/article/viewFile/212/128>. Acesso em: 11 jan. 2010.

VILPOUX, O.F. Competitividade da mandioca no Brasil, como matéria prima para amido. Informações Econômicas, v.38, p.27-38, 2008. Disponível em: <ftp://ftp.sp.gov.br/ftpiea/ publicacoes/tec3-1108.pdf $>$. Acesso em: 11 jan. 2010.

ZACARIAS, A.M. et al. Characterization and genetic distance analysis of cassava (Manihot esculenta Crantz) germplasm form Mozambique using RAPD fingerprinting. Euphytica, v.138, p.49-53, 2004. Disponível em: <http://www.springerlink.com/ content/r9470q13461745n7/fulltext.pdf>. Acesso em: 11 jan. 2010. doi: 10.1023/B:EUPH.0000047057.05642.74. 